\title{
Collisional disruption of weakly sintered porous targets at low-impact velocities
}

\author{
Masato Setoh $^{1}$, Akiko M. Nakamura ${ }^{1}$, Naru Hirata $^{2}$, Kensuke Hiraoka $^{1}$, and Masahiko Arakawa ${ }^{3}$ \\ ${ }^{1}$ Graduate School of Science and Technology, Kobe University, 1-1 Rokkodai-cho, Nada-ku, Kobe 657-8501, Japan \\ ${ }^{2}$ Department of Computer Software, Aizu University, Ikki-machi, Aizu-Wakamatsu City, Fukushima 965-8580, Japan \\ ${ }^{3}$ Graduate School of Environmental Studies, Nagoya University, Furo-cho, Chikusa-ku, Nagoya 464-8601, Japan
}

(Received April 14, 2006; Revised October 16, 2006; Accepted December 26, 2006; Online published May 7, 2007)

\begin{abstract}
Porous structure is common in the asteroids and satellites of the outer planets. In order to study the relationship between the structure of small bodies and their thermal and collisional evolution, we performed impact disruption experiments on porous sintered targets using a light-gas gun at velocities ranging from 10 to $100 \mathrm{~m} / \mathrm{s}$. The sintered glass bead targets were prepared to have roughly the same porosity but with different compressive strengths, ranging over an order of magnitude, by controlling sintering duration and temperature. The results of the impact experiments show that the targets of higher compressive strength have higher impact strengths. However, compared to previous results on impact disruption of porous sintered targets with a collisional velocity of approximately $6 \mathrm{~km} / \mathrm{s}$, the values of impact strength in this study were found to be lower by an order of magnitude.
\end{abstract}

Key words: Porosity, impact, small body, asteroid.

\section{Introduction}

Since the end of the accretion phase of the Solar System approximately $4.5 \mathrm{Gyr}$ ago, collisional disruptions have played various roles in the formation and evolution processes of the planetary system. The major collisional disruption zones are the main asteroid belt (Davis et al., 1989; Weidenschilling et al., 2001) and the Edgeworth-Kuiper (EK) belt (Farinella and Davis, 1996). The outcomes of collisional disruptions have been studied for decades by laboratory experiments, scaling approaches, and numerical simulations (Fujiwara et al., 1989; Holsapple et al., 2002). In the earliest studies, the materials used in laboratory experiments were mostly non-porous rocks and ices. However, porous structure has since been found to be common among asteroids (e.g., Britt et al., 2002; Fujiwara et al., 2006) and, consequently, the outcomes of collisional disruption of porous bodies have become of great significance for studying the origins and collisional evolution of small bodies.

The threshold value of energy density (which is the kinetic energy of the colliding bodies per the mass of the larger body or the total mass of the system, $Q$ ) for catastrophic disruption, $Q^{*}$, is defined as the energy for which the largest remaining intact piece has one half of the mass of the original. $Q^{*}$ generally depends on material strength (Holsapple et al., 2002); however, porosity plays a complicated role. The static compressive strength (S) of porous material is usually lower than that of dense material, as indicated by an empirical formula known for ceramics (Ryshkewitch 1953), given by $S=c_{1} \exp \left(-c_{2} P\right)$, where $c_{1}$ and $c_{2}$

Copyright (c) The Society of Geomagnetism and Earth, Planetary and Space Sciences (SGEPSS); The Seismological Society of Japan; The Volcanological Society of Japan; The Geodetic Society of Japan; The Japanese Society for Planetary Sciences; TERRAPUB are constants, and $P$ denotes porosity. On the one hand, with increasing porosity, the target body becomes weaker; on the other hand, the increasing volume of void space decreases the transmission efficiency of stress wave in the target body so that porous bodies appear to be strong against impact.

An impact experiment by Love et al. (1993) using sintered glass beads at high impact velocities produced an empirical relationship whereby targets with higher compressive strength have higher $Q^{*}$ and targets with higher porosity have higher $Q^{*}$. In their experiment, the porosity of the targets was varied without fixing the compressive strength. The results suggested that targets with $5 \%$ porosity have a 140-fold higher compressive strength than targets with $60 \%$ porosity, but they only have 0.4 -fold $Q^{*}$ of those of the much more porous targets.

Although the average collisional velocity in the main asteroid belt is approximately $5 \mathrm{~km} / \mathrm{s}$ (Bottke et al., 1994), the mean collisional velocity in the E-K belt is less than approximately $1 \mathrm{~km} / \mathrm{s}$ (Davis and Farinella, 1997). As suggested by previous studies (Takagi et al., 1984; Ryan et al., 1999), the conclusions drawn from high-velocity impact experiments cannot simply be applied to impacts at low velocities. For example, the peak pressure at an impact does not scale in a straightforward fashion with $Q$, because the dependence of the peak pressure at impact velocity is approximately linear under low-velocity conditions but quadratic at extremely high velocities. Takagi et al. (1984) introduced another scaling parameter, $P I$, instead of $Q$, for characterizing collisional experiments of different projectile and target materials and impact velocities. The scaling parameter, called the non-dimensional impact stress (NDIS) is given in the form of $P I=P_{0}(L / R)^{3} / Y$, where $P_{0}=$ peak shock 
pressure, $L=$ projectile size, $R=$ target size, and $Y=$ material strength of the target where compressive strength $S$ was used in practice.

Another difference between low- and high-velocity collisions is that of the ratio of the sizes of projectile and target. A lower velocity impact needs a larger mass of projectile to attain the same energy density $(Q)$ or NDIS $(P I)$ than a higher velocity impact. In such a lower impact velocity case, the stress wave does not travel sufficient distance to become attenuated, and it is expected that porosity plays a rather minor role in the impact disruption. Ryan et al. (1999) conducted low-velocity impact experiments at a few hundreds of meters per second using ice projectiles and porous ice targets. Based on their results, they suggested that the $Q^{*}$ of porous bodies is higher by a factor of about 5 than the values inferred for aluminum projectiles and solid ice targets. In other words, less porous targets have an impact strength only 0.2 -fold that of the porous targets. Ata first glance, this may appear to conflict with the expectation suggested above (a lesser role for porosity in low-velocity impacts than in high-velocity impacts). However, a definitive quantitative comparison should wait until the target and projectile properties, such as compressive strength, have been characterized.

Our goal is to understand the nature of small bodies in the solar system, i.e., the rubble pile hypothesis and the effect of porous structure on their collisional evolution. Several potentially important parameters control the outcome of the impact disruption of porous bodies, as described above. Systematic impact experiments with porous targets are required to establish a database that can serve as a useful reference for numerical modeling. However, the existing differences in multiple parameters between different experiments prevented us from directly comparing one result with others. Therefore, we carried out a new series of impact disruption experiments with porous targets in which we varied-independently - the impact velocity, the compressive strength of the targets, and the porosity. In this study, we kept the porosity constant and focused on the effect of static strength. Section 2.1 explains the method of target preparation and the target properties. Measurements of compressive strength are described in Section 2.2 and those of their impact strength, $Q^{*}$, measured by a light-gas gun, are described in Section 2.3. For estimating the possible effect of secondary fragmentation on $Q^{*}$, we performed free fall experiments to simulate the collisions between the fragments and the wall of the chamber in Section 2.4. Finally, in Section 3, we discuss these results and report the differences in the effect of porosity on impact strength for low-and high-velocity impacts.

\section{Experiments}

\subsection{Target manufacture and properties}

To produce porous targets, we sintered soda lime glass beads (diameter: $50 \mu \mathrm{m}$; nominal density: $2.5 \mathrm{~g} / \mathrm{cm}^{3}, \mathrm{Fu}-$ jikihan, Japan) (Love et al., 1993; Michikami, 2007). The sintering process consisted of pouring the glass beads into a commercial tea cup-shaped mold (diameter at top: $4.0 \mathrm{~cm}$; diameter at bottom: $3.0 \mathrm{~cm}$; depth: $3.0 \mathrm{~cm}$ ) and then positioning the mold at the center of an oven which was sub-
Table 1.

\begin{tabular}{cccccc}
\hline $\begin{array}{c}\text { Target } \\
\text { type } \\
\left({ }^{\circ} \mathrm{C}\right)\end{array}$ & $\begin{array}{c}\text { Sintering } \\
\text { temperature } \\
(\mathrm{min})\end{array}$ & $\begin{array}{c}\text { Sintering } \\
\text { duration }\end{array}$ & $\begin{array}{c}\text { Density } \\
\left(\mathrm{g} / \mathrm{cm}^{3}\right)\end{array}$ & $\begin{array}{c}\text { Porosity } \\
(\%)\end{array}$ & $\begin{array}{c}\text { Strength } \\
(\mathrm{MPa})\end{array}$ \\
\hline $\mathrm{A}$ & 660 & 30 & 1.55 & $37.7 \pm 0.8$ & $2.76 \pm 0.88$ \\
$\mathrm{~B}$ & 650 & 30 & 1.48 & $40.7 \pm 0.9$ & $1.01 \pm 0.07$ \\
$\mathrm{C}$ & 640 & 30 & 1.40 & $43.9 \pm 0.8$ & $-\mathrm{a}$ \\
$\mathrm{D}$ & 630 & 30 & 1.35 & $45.7 \pm 2.5$ & $-\mathrm{a}$ \\
$\mathrm{E}$ & 650 & 20 & 1.46 & $41.5 \pm 0.8$ & $-\mathrm{a}$ \\
$\mathrm{F}$ & 650 & 10 & 1.40 & $43.8 \pm 0.8$ & $-\mathrm{a}$ \\
\hline
\end{tabular}

${ }^{\mathrm{a}}$ Too fragile to extract cores (see text).

Table 2.

\begin{tabular}{cccccc}
\hline $\begin{array}{c}\text { Target } \\
\text { type } \\
\left({ }^{\circ} \mathrm{C}\right)\end{array}$ & $\begin{array}{c}\text { Sintering } \\
\text { temperature } \\
(\mathrm{min})\end{array}$ & $\begin{array}{c}\text { Sintering } \\
\text { duration }\end{array}$ & $\begin{array}{c}\text { Density } \\
\left(\mathrm{g} / \mathrm{cm}^{3}\right)\end{array}$ & $\begin{array}{c}\text { Porosity } \\
(\%)\end{array}$ & $\begin{array}{c}\text { Strength } \\
(\mathrm{MPa})\end{array}$ \\
\hline $\mathrm{A}^{\prime}$ & 660 & 30 & 1.48 & $40.9 \pm 0.8$ & $7.8 \pm 2.4$ \\
$\mathrm{~B}^{\prime}$ & 650 & 30 & 1.47 & $41.4 \pm 0.8$ & $3.8 \pm 1.3$ \\
$\mathrm{C}^{\prime}$ & 640 & 30 & 1.44 & $42.5 \pm 2.1$ & $2.6 \pm 0.5$ \\
$\mathrm{D}^{\prime}$ & 630 & 30 & 1.39 & $44.3 \pm 1.3$ & $0.3 \pm 0.14$ \\
$\mathrm{E}^{\prime}$ & 650 & 20 & 1.52 & $39.3 \pm 0.8$ & $1.7 \pm 0.66$ \\
$\mathrm{~F}^{\prime}$ & 650 & 10 & 1.47 & $41.3 \pm 0.8$ & $0.2 \pm 0.08$ \\
\hline
\end{tabular}

sequently heated from room temperature to maximum temperatures of $630,640,650$, and $660^{\circ} \mathrm{C}$ in a 25 -min interval. The length of the baking process at the maximum temperature was $30 \mathrm{~min}$, at which point the heater was switched off. The oven was not opened for about $10 \mathrm{~h}$, until the internal temperature had returned to room temperature. This long cooling time was employed to minimize possible internal thermal stresses in the targets. We produced other targets by raising their temperature from room temperature to $650^{\circ} \mathrm{C}$ during a 25-min interval and maintaining the baking temperature at $650^{\circ} \mathrm{C}$ for 10 or $20 \mathrm{~min}$. The target properties are summarized in Table 1.

Our aim was to obtain target types with similar densities but different strengths. Their porosities were approximately $40 \%$, which is similar to those of porous asteroids such as the C-type average (Britt et al., 2002). The targets were white, and their constituent beads were still identifiable.

\subsection{Strength testing}

One copy of each target type was used for unconfined compressive strength testing at Kobe University. Several cylindrical cores (height: $1.5 \mathrm{~cm}$; diameter: $1.0 \mathrm{~cm}$ ) were drilled from each of the A and B targets, with their axes parallel to that of the cup-shaped target. The cores were placed in a compression testing machine, which provided records of the force applied and the displacement of the moving crosshead. The strain rate was $1.1 \times 10^{-3} \mathrm{MPa} / \mathrm{s}$. The unconfined compressive strength values thus obtained are presented in Table 1 . We could not extract any cores from the C-, D-, E-, and F-type targets because they were too fragile to be drilled. We then constructed cylindrical targets (diameter: $1.0 \mathrm{~cm}$; depth: $1.5 \mathrm{~cm}$ ) using a smaller cylindrical mold, but used the same sintering conditions as those for the cup-shaped mold. The densities and porosities of these targets are shown in Table 2. The compressive strength values reported in Table 2 are about 2.8- and 3.8- 

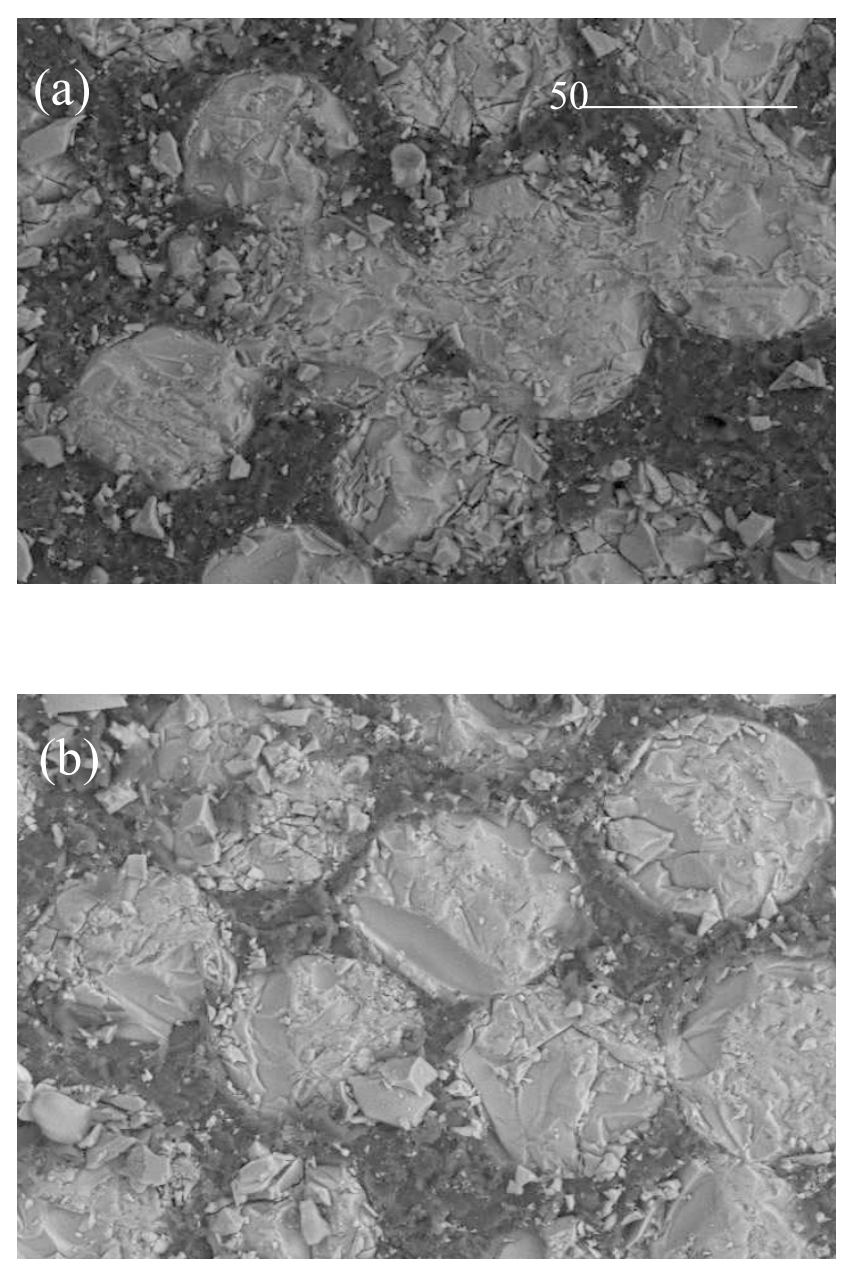

Fig. 1. The microscope images of (a) type- $\mathrm{A}^{\prime}$ and (b) $-\mathrm{D}^{\prime}$ targets.

fold higher than those in Table 1. We infer that this is due to the smaller heat capacity of the mold filled with glass beads for the samples of Table 2, as compared to those of Table 1. The sintering process was able to progress further for the samples in Table 2 due to their smaller heat capacity. Figure 1(a) and (b) shows electron micrographs of the $\mathrm{A}^{\prime}$ and $\mathrm{D}^{\prime}$ targets in Table 2. The necks in target $\mathrm{A}^{\prime}$ are more developed due to the higher maximum temperature.

Figure 2(a)-(c) shows the stress-strain curves of the B, $\mathrm{B}^{\prime}$, and $\mathrm{D}^{\prime}$ targets. Samples of the A-, $\mathrm{A}^{\prime}-, \mathrm{B}-, \mathrm{B}^{\prime}-$, $\mathrm{C}^{\prime}$-, and $\mathrm{E}^{\prime}$-type targets showed well-determined breaking points. Despite their weak cohesion and tendency to compress, these targets broke decisively along a vertically plane through the center. The fracture surfaces were different from those observed in Love's (1993) and Michikami's (2007) experiments. In contrast, the $\mathrm{D}^{\prime}$ - and $\mathrm{F}^{\prime}$-type targets did not break decisively. These samples were broken stepwise and their breaking points were not clear. We regarded the maximum point of the stress-strain curve as their compressive strength.

\subsection{Impact experiments}

The impact experiments were carried out in air using a light-gas gun at Kobe University. For the six different target types, we used cylindrical polycarbonate projectiles that were $1.5 \mathrm{~cm}$ high and $1.0 \mathrm{~cm}$ in diameter.

Each target was put on a cylindrical stand that was 10
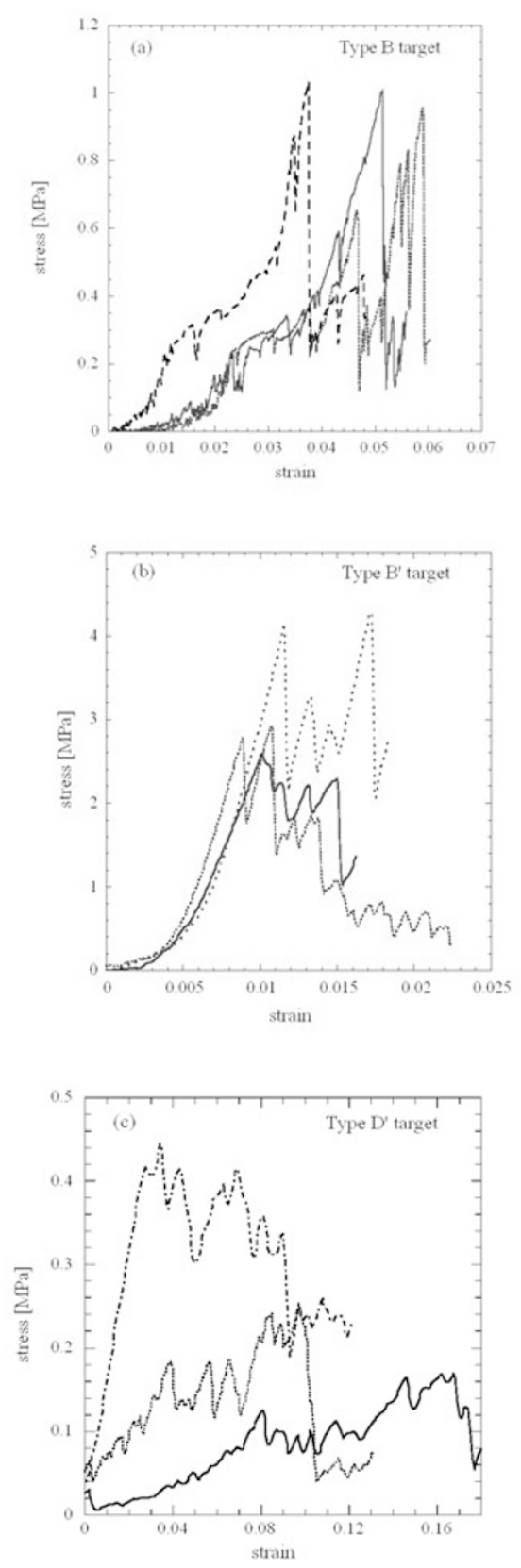

Fig. 2. Relation between the stress and the strain of (a) type-B, (b) - $\mathrm{B}^{\prime}$ and (c) $-\mathrm{D}^{\prime}$ targets. The three curves in each figure correspond to three specimens.

$\mathrm{cm}$ high and situated $10 \mathrm{~cm}$ from the muzzle of the gun. The accuracy of target positioning was within $3 \mathrm{~mm}$. A few cellophane sheets were used as diaphragms to regulate the speed of the projectiles, which was usually measured using high-speed video images taken with a high-speed camera (Photron FASTCAM-PCI) at 2,000-5,000 fps; the speed varied between 10 and $100 \mathrm{~m} / \mathrm{s}$. When the high-speed video was not available, we measured the time of flight of the projectile using thin aluminum foil strips. The typical measurement error was $10 \%$. The velocities of ejecta from a typeB target were measured on images taken at 30,000 fps by a different high-speed camera (Photron FASTCAM-APX RS). Figure 3 shows an example of the sequence of collisional disruption. 

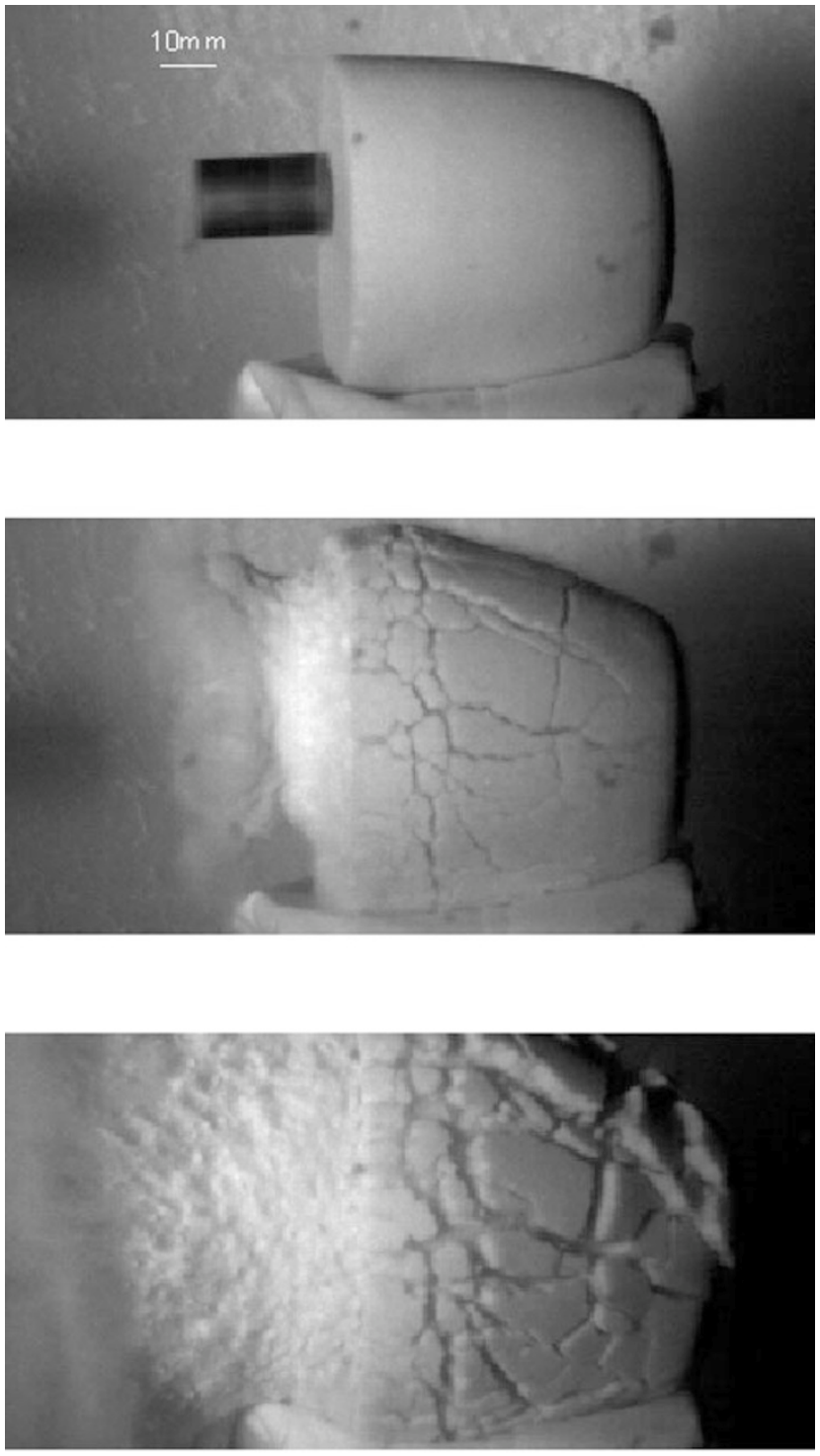

Fig. 3. High-speed camera images of the impact experiment against the type-B target with an impact velocity of $100 \mathrm{~m} / \mathrm{s}$ taken at 30,000 fps. Note that the result of this shot is not included in Fig. 4. (a) The second frame from the contact between the projectile and the target, (b) the 22nd frame, (c) the 72nd frame.

\subsection{Free fall experiments}

In this type of impact experiments, secondary fragmentation (Nakamura and Fujiwara, 1991) may occur. Ejecta from the targets can hit the wall of the chamber, at which point the ejecta may break into smaller pieces. The mass of the largest fragment mass may decrease by this process; accordingly, the impact strength $Q^{*}$ may be estimated to be lower than the true value. In the case of type-B targets with an impact velocity of about $100 \mathrm{~m} / \mathrm{s}$, the maximum fragment velocities determined by the high-speed camera images were less than $1 \mathrm{~m} / \mathrm{s}$ for a $1-\mathrm{cm}(1-2 \mathrm{~g})$ particle and a few meters per second for much smaller particles. On the other hand, the free fall velocity from the target stand was $1.4 \mathrm{~m} / \mathrm{s}$. Therefore, the minimum impact velocity of the ejecta against the walls was $1.4 \mathrm{~m} / \mathrm{s}$. A previous study of low-velocity collisions of centimeter-scale blocks showed that ice begins to fracture at several meters per sec-

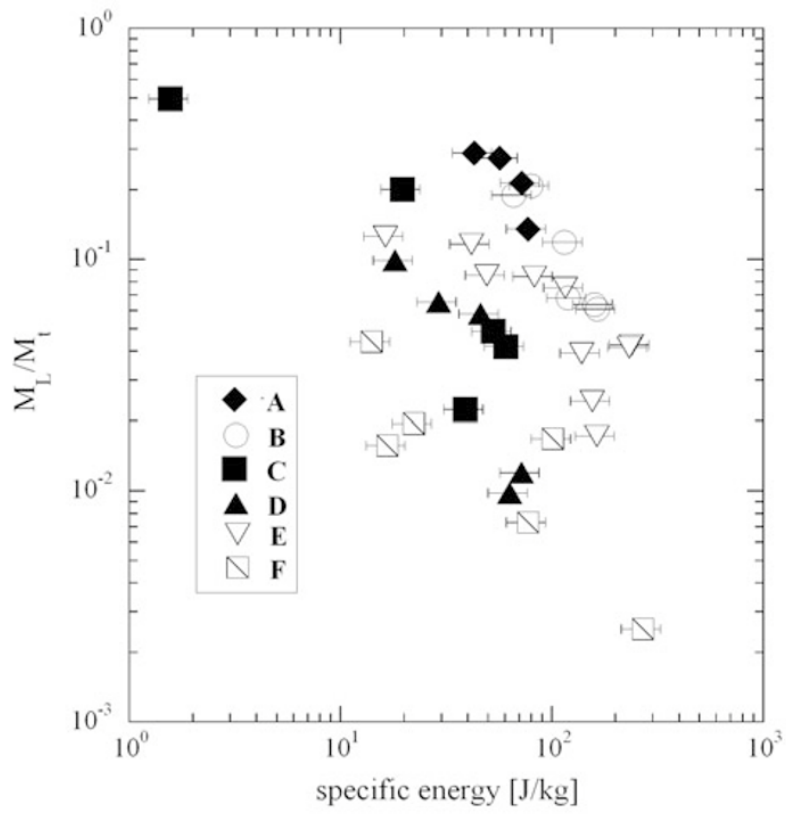

Fig. 4. The ratio of the largest fragment mass $\left(M_{L}\right)$ to target mass $\left(M_{t}\right)$ versus the specific kinetic energy of the projectile per target mass.

ond, whereas rock, which is much stronger than ice, begins to fracture at a few tens of meters per second (Hartmann, 1978). Since the strength of our targets was similar to or less than that of ice, secondary fragmentation was expected to occur for our targets.

We thus performed very low velocity impact experiments using type-B and -D targets. We dropped the fragments onto one of the acrylic windows of the chamber at velocities of up to a few meters per second.

\section{Results and Discussion}

For most of the impacts, the projectiles remained almost intact, and only in the case of some of the higher velocities were their shapes deformed. Figure 4 shows the ratio of the mass of the largest fragment $\left(M_{L}\right)$ to that of the target $\left(M_{t}\right)$ versus the specific kinetic energy of the projectile per target mass, as an indicator of the degree of fragmentation (Fujiwara et al., 1989). We determined the value of $Q^{*}$ for each target type by fitting a power law to the data in each case.

Figure 5 shows the relationship between $Q^{*}$ and the compressive strength of the target. The empirical relation is given by

$$
Q *=(4.2 \pm 2.1) S^{1.00 \pm 0.37}
$$

when the strength values in Table 2 are used, whereas a relation of $Q *=95.3 S^{0.45}(1-P)^{-3.6}$ was previously obtained (Love et al., 1993). In order to compare the absolute values of $Q^{*}$, we substituted a typical value of $S=2(\mathrm{MPa})$ and $P=0.40$, which gave a value of 8.4 for our results and a value of 819 for the relationship given by Love et al. (1993). Possible reasons for the very low values of $Q^{*}$ in the present results may be: (1) the lower the impact velocity, the larger the mass of the projectile needs to be in order to attain the same energy density $(Q)$ as a higher impact velocity and, therefore, a smaller degree of attenuation of the 


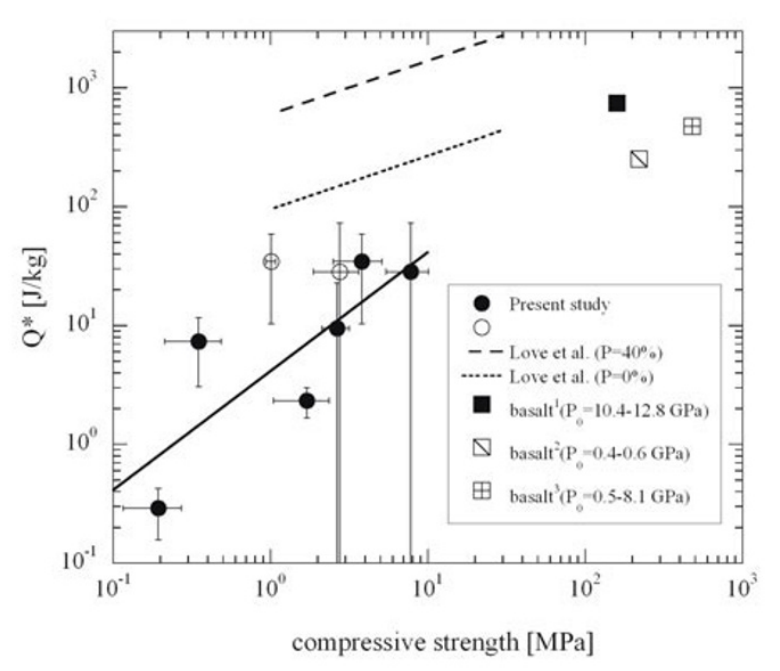

Fig. 5. Relation between the compressive strength and $Q^{*}$ of all target types. Filled circles show the strength data from Table 2, while open circles show those from Table 1 . The solid line is a fit to the data points of the filled circles. The dashed line shows the empirical relation for targets with $40 \%$ porosity derived by Love et al. (1993). The dotted line shows the relation for $0 \%$ porosity. Basalt data were based on previous studies; 1: Fujiwara et al. (1977) with impact velocity of 2500-2900 $\mathrm{m} / \mathrm{s}, 2$ : Matsui et al. (1982) with $50-150 \mathrm{~m} / \mathrm{s}$, 3: Takagi et al. (1984) with $70-990 \mathrm{~m} / \mathrm{s}$. The initial peak pressure, $P_{0}$, of each experiment is shown in parenthesis.

stress wave in the targets, despite the porosity, due to the small contrast in size between the projectiles and targets; (2) difference in the dependence of the initial peak pressure on impact velocity between the high- and low-velocity impact disruption; (3) breakup of the largest fragments (secondary fragmentation) when they hit the wall of the chamber. With respect to hypothesis (3), Fig. 6(a) and (b) shows the results of the dropping tests with the type-B and -D targets, respectively. The ejection velocities of the fastest fragments from the type-B target described in Section 2.4 are also shown. In this study, any piece that was found to be separated from the original piece upon impact on the plate is referred to as "broken." A previous collision experiment using ice spheres and an ice surface found a systematic size dependence of the threshold velocity for the onset of fracture (Higa et al., 1998), and the size dependence was attributed to the strainrate fracture strength. In accordance with these earlier results, we also found size dependences for both types of target. The threshold "broken" velocity of a given mass shown in Fig. 6(a) and (b) is larger than the maximum ejection velocity of the ejecta of a given mass. Figure 6(b) shows that the largest samples of the type-D target broke at free fall velocity $(1.4 \mathrm{~m} / \mathrm{s})$ from the target stand. Even then, however, the largest fragment was more than half the size of the original. Accordingly, the blocks hitting the acrylic plate may break marginally; however, the large difference in the absolute values of $Q^{*}$ between the previous work and the present results cannot be explained by the secondary fragmentation.

The diameter ratio of the target to the projectile for our experiments was only approximately 3 , and the projec-
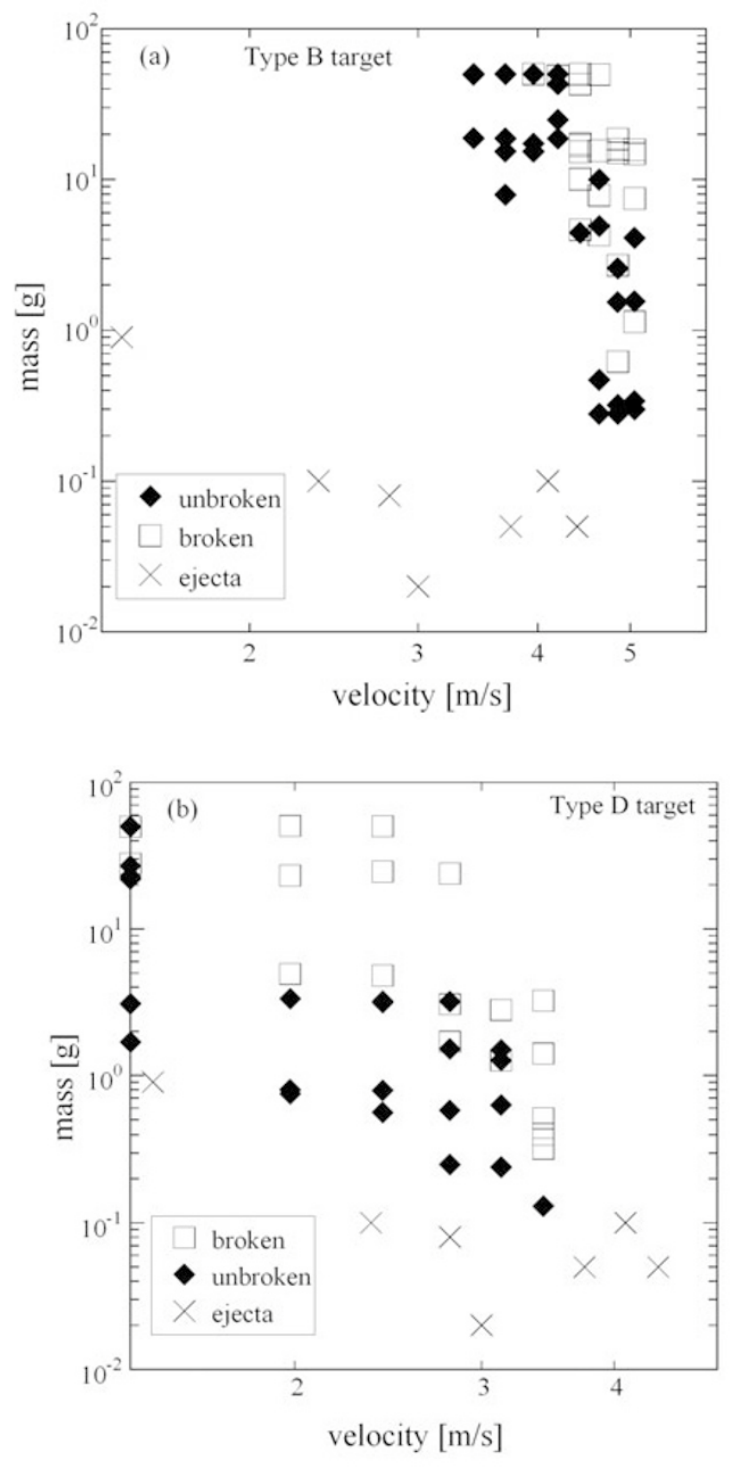

Fig. 6. The results of free fall experiments of type-B and -D targets. Ejection velocities measured for the fastest fragments from the shot shown in Fig. 3 are also plotted.

tile energy was expected to be transferred more effectively throughout the whole target, in contrast to the earlier experiments by Love et al. (1993) in which the diameter ratio was larger by approximately one order of magnitude than in our experiments. Thus, the difference in the impact strength $Q^{*}$ between the two experiments was probably partly due to hypothesis (1) - the difference in the degree of attenuation of the stress wave in the target-as expected.

However, hypothesis (1) does not fully explain the large difference because the values of $Q^{*}$ in this study are even lower than the values expected for non-porous glass targets, based on the equation of Love et al. (1993); the difference is of a few factors, as shown in Fig. 5. Accordingly, hypothesis (2) also appears to play a role. Since the Hugoniot parameters of the sintered glass beads were not available, we could not examine our results using NDIS. Instead, in Fig. 5 we provide previous results for basalt fragmentation under different impact conditions in order to illustrate differences in $Q^{*}$ between high-velocity (or high initial pressure) im- 
pacts and low-velocity impacts. The basalt data show that the values of $Q^{*}$ from the experiments with higher initial pressure (in this case, this is equivalent to having a higher impact velocity) are about threefold higher than those with lower initial pressure (or lower impact velocity). A similar tendency can be expected for the fragmentation of sintered glass bead targets.

\section{Summary}

We succeeded in producing sintered glass bead targets that had roughly the same porosity but different compressive strength. The results of impact experiments using a light-gas gun showed that the targets with a higher compressive strength had a higher impact strength. However, the values of the impact strength found in this study were lower than those reported in previous experiments by more than an order of magnitude. We conducted low-velocity impact tests of the fragments to examine the degree of secondary fragmentation expected to occur when the fragments hit the walls of the experimental chamber. The results showed that marginal secondary fragmentation may occur but that it is not severe enough to explain the much lower impact strength that we observed. The difference in impact strength between our results and those of previous experiments is probably due to differences in impact velocity and the degree of attenuation of the stress wave in the target.

The study of collisional disruption of porous bodies at impact velocities of less than $1 \mathrm{~km} / \mathrm{s}$ has become increasingly important for understanding the collisional evolution of bodies in the outer solar system, namely the E-K belt and beyond, where the number of detected bodies is increasing. The static strength and porosity of these bodies is expected to be highly dependent upon their collisional and thermal history. The present study gives an initial report of a new series of experiments aimed at achieving a clearer view of how collisional outcomes are dependent upon static strength and impact velocity.

Acknowledgments. We thank K. Tanaka of Kobe University for his support in making the cores for compression testing. We also thank Y. Wada and N. Ishizaki for considerable support during the SEM observations and K. Sangen for his preparation of the projectiles. We are grateful to S. Nakatsubo of the Institute of Low Temperature Science, Hokkaido University, for manufacturing the gas-gun. The chance to test the high-speed camera (FASTCAMAPX RS) was offered by Photron and conducted by ourselves. This work was supported by the "21st Century COE Program of Origin and Evolution of Planetary Science" of the Ministry of Education, Culture, Sports, Science and Technology (MEXT), Japan.

\section{References}

Britt, D. T., D. Yeomans, K. Housen, and G. Consolmagno, Asteroid density, porosity, and structure, in Asteroids III, edited by W. F. Bottke, Jr. et al., 485 pp., University of Arizona Press, Tucson, 2002.

Bottke Jr., W. F., et al., Velocity distributions among colliding asteroids, Icarus, 107, 255-268, 1994.

Davis, D. R., et al., Asteroid collisional history: Effects on sizes and spins. in Asteroids II, edited by R. P. Binzel, T. Gehrels, and M. Matthews, pp. 805-826, Univ. of Arizona Press, Tucson, 1989.

Davis, D. R. and P. Farinella, Collisional evolution of Edgeworth-Kuiper belt objects, Icarus, 125, 50-60, 1997.

Farinella, P. and D. R. Davis, Short-period comets: Primordial bodies or collisional fragments?, Science, 273, 938-941, 1996.

Fujiwara, A., G. Kamimoto, and A. Tsukamoto, Destruction of basaltic bodies by high velocity impact, Icarus, 31, 277-288, 1977.

Fujiwara, A., P. Cerroni, D. Davis, E. Ryan, and M. Di Martino, Experiments and Scaling laws for catastrophic collisions, in Asteroids II, edited by R. P. Binzel et al., 240 pp., University of Arizona Press, 1989.

Fujiwara, A., et al., The rubble-pile asteroid Itokawa as observed by Hayabusa, Science, 312, 1330-1334, 2006.

Hartmann, W. K., Planet formation - mechanism of early growth, Icarus, 33, 50-61, 1978.

Higa, M., M. Arakawa, and N. Maeno, Size dependence of restitution coefficients of ice in relation to collision strength, Icarus, 133, 310-320, 1998.

Holsapple, K., I. Giblin, K. Housen, A. Nakamura, and E. Ryan, Asteroid impacts: laboratory experiments and scaling laws, in Asteroids III, edited by W. F. Bottke, Jr. et al., 443 pp., University of Arizona Press, Tucson, 2002.

Love, S. G., F. Hörz, and D. E. Brownlee, Target porosity effects in impact cratering and collisional disruption, Icarus, 105, 216-224, 1993.

Matsui, T., T. Waza, K. Kani, and S. Suzuki, Laboratory simulation of planetesimal collisions, J. Geophys. Res., 87, 10,968-10,982, 1982.

Michikami, T., K. Moriguchi, S. Hasegawa, and A. Fujiwara, Ejecta velocity distribution for impact cratering experiments on porous and low strength targets, Planet. Space Sci., 2007.

Nakamura, A. and A. Fujiwara, Velocity distribution of fragments formed in a simulated collisional disruption, Icarus, 92, 132-146, 1991.

Ryan, E. V., D. R. Davis, and I. Giblin, A laboratory impact study of simulated Edgeworth-Kuiper Belt objects, Icarus, 142, 56-62, 1999.

Ryshkewitch, E., Compression strength of porous sintered alumina and zirconia, J. Am. Ceram. Soc., 36, 65-68, 1953.

Takagi, Y., H. Mizutani, and S.-I. Kawakami, Impact fragmentation experiments of basalts and pyrophyllites, Icarus, 59, 462-477, 1984.

Weidenschilling, S. J., D. R. Davis., and F. Marzari., Very early collisional evolution in the asteroid belt, Earth Planets Space, 53, 1093-1097, 2001 .

M. Setoh (e-mail: setoh@kobe-u.ac.jp), A. M. Nakamura, N. Hirata, K. Hiraoka, and M. Arakawa 